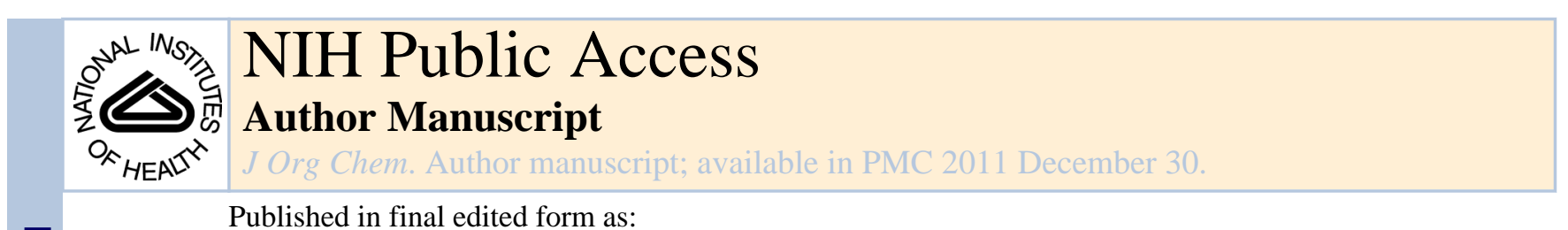

J Org Chem. 2005 September 16; 70(19): 7711-7714. doi:10.1021/jo0511039.

\title{
Opening of Aryl-Substituted Epoxides to form Quaternary Stereogenic Centers: Synthesis of (-)-Mesembrine
}

\author{
Douglass F. Taber ${ }^{\star}$ and Yigang He \\ Department of Chemistry and Biochemistry, University of Delaware, Newark, DE 19716
}

\begin{abstract}
Cycloalkanones are easily converted into aryl-substituted cyclic alkenes by the addition of an aryl Grignard reagent followed by dehydration. These alkenes are good substrates for asymmetric epoxidation. We have found that the addition of allylic and benzylic Grignard reagents can occur preferentially at the benzylic position of the derived epoxides, to give the quaternary stereogenic center. This approach led to a short synthesis of the nanomolar serotonin re-uptake inhibitor (-)mesembrine.
\end{abstract}

\section{Introduction}

Alicyclic rings containing quaternary stereogenic centers ${ }^{1}$ with attached aromatic rings are a common motif both among physiologically-active alkaloids, including mesembrine $\mathbf{1}$ and morphine $\mathbf{2}$, and in medicinal chemistry. We report what promises to be a general enantioselective method for the construction of such stereogenic centers. We have illustrated the power of this approach with a short synthesis of (-)-mesembrine $1 .^{2-4}$<smiles>COc1ccc(C23CCC(=O)C[C@H]2N(C)CC3)cc1OC</smiles>

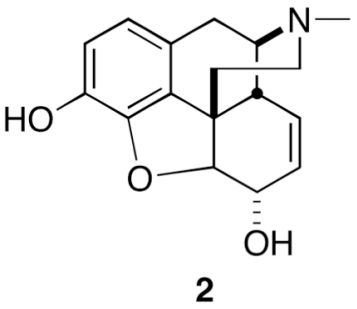

(-)-Mesembrine $\mathbf{1}$ was isolated as the major alkaloid component of Sceletium tortuosum, chewed for its stimulant properties by the indigenous population of the Kalahari. In a limited trial, ${ }^{5}$ the dried and powdered plant preparation, a mild stimulant, was shown to have marked anxiolytic properties. This crude preparation was also shown to be a powerful antiaddictive. The pure alkaloid 1 was recently shown ${ }^{6}$ to be a nanomolar inhibitor of serotonin re-uptake, with efficacy being observed at a once-a-day oral dose of 100 micrograms.

*Taberdf@udel.edu.

This work is dedicated to the memory of Conrad J. Kowalski, a master of the science and art of organic synthesis.

SUPPORTING INFORMATION. General experimental details, characterization of the products from Table 1, and spectra for all new compounds and for $\mathbf{1}$. This material is available free of charge via the internet at http://pubs.acs.org. 


\section{Results and Discussion}

We recently observed (Scheme 1$)^{7}$ that allylic and benzylic Grignard reagents would open epoxides such as $\mathbf{3}$ to give selectively the product $\mathbf{4}$. We envisioned that an epoxide such as 5, with an attached arene, ${ }^{8}$ might be similarly activated.

In the event (Table 1), we were not disappointed. Even a simple non-activating arene ${ }^{9-11}$ (entry 1) gave a substantial portion of the desired product. An activating $p$-methyl (entry 2) increased the fraction of the desired secondary alcohol. With the still more activating $p$ methoxy (entry 3 ), the cyclic quaternary stereogenic center was formed smoothly.

We also explored alternative nucleophiles with the epoxide $\mathbf{5} \mathbf{d}^{10}$ (Table 1). We found that allylic and benzylic Grignard reagents gave the desired ring-opening products. It was striking that although the alkyne-activated epoxide (Scheme 1) gave the branched product 4 with crotyl magnesium chloride, the arene-activated epoxide gave only the complementary linear product 6g. The less reactive and more basic alkyl, phenyl and vinyl Grignard reagents gave primarily undesired side products. This difference is attributable at least in part to the lack of reactivity of these nucleophiles toward the trisubstituted cyclic expoxide. $\mathrm{Et}_{3} \mathrm{Al}^{8 \mathrm{~d}}$ gave rearrangement to the cyclohexanone followed by hydride reduction, while $\mathrm{Et}_{2} \mathrm{AlCN}^{12}$ gave only rearrangement to the ketone.

The beauty of this approach is that it allows the construction of a quaternary stereogenic center on an existing ring. It is particularly exciting that using the Shi epoxidation, ${ }^{13}$ one can convert a prochiral cyclic alkene to the enantiomerically-defined product. To establish the viability of this approach, and to confirm the sense of absolute stereocontrol of the process, we converted the monoprotected cyclohexanone 6 (Scheme 2) to (-)-mesembrine (1).

The cyclohexanone 8 reacted with the aromatic Grignard reagent 9 to give the known ${ }^{10}$ alkene 10, after dehydration using PTSA in the presence of excess ethylene glycol. Shi expoxidation ${ }^{13}$ of $\mathbf{1 0}$ followed by ring-opening of the resulting crude epoxide by allylmagnesium chloride gave the enantiomerically enriched secondary alcohol $\mathbf{6 d}{ }^{10}$ The enantiomers of $\mathbf{6 d}$ were well resolved by chiral HPLC (Chiralcel OD, 15\% isopropanol / hexane, $1 \mathrm{~mL} / \mathrm{min}$, retention time: $17.6 \mathrm{~min}$ for the minor enantiomer and $34.2 \mathrm{~min}$ for the major enantiomer). The alcohol 6d derived from Shi epoxidation had $96 \%$ ee.

Exposure of $\mathbf{6 d}$ to $10 \%$ aqueous $\mathrm{HCl}$ in THF gave the enone $11 .{ }^{4 \mathrm{~b}}$ Selective ozonolysis of the terminal double bond in $\mathbf{1 1}$ followed by treatment ${ }^{14}$ of the resulting ozonide in situ with $\mathrm{NaBH}_{4}$ in the presence of $\mathrm{CeCl}_{3}{ }^{15}$ furnished the diol $\mathbf{1 1}^{3 \mathrm{~h}}$ as a $4: 1$ mixture of diastereomers. The primary hydroxyl group in $\mathbf{1 1}$ was selectively converted into the corresponding tosylate 12, which on heating with $40 \%$ aqueous methylamine followed by oxidation using activated $\mathrm{MnO}_{2}{ }^{3 \mathrm{~h}}$ gave (-)- mesembrine $(\mathbf{1}),\left([\alpha]^{22} \mathrm{D}=-55.4\right.$, lit. $=-62.8,{ }^{3 \mathrm{a}}-63.3^{3 \mathrm{~d}},-53.0,{ }^{3 \mathrm{e}}$ $-59.3,3 \mathrm{~h})$, identical $\left({ }^{1} \mathrm{H},{ }^{13} \mathrm{C}\right.$ NMR $)$ with authentic material.

\section{Conclusion}

We believe that the approach outlined here will be a useful strategy for the enantioselective assembly of cyclic quaternary stereogenic centers having attached aromatic rings, complementary to such recently-developed methods as enantioselective allylation ${ }^{16}$ and enantioselective arylation. ${ }^{17}$ 


\section{Experimental}

\section{Epoxide (5c)}

A solution of 1-(4-methoxyphenyl)-cyclohexene $(0.752 \mathrm{~g}, 3.68 \mathrm{mmol})$ in $2 \mathrm{~mL}$ of acetone was added to a solution of dimethyldioxirane ${ }^{4}(50 \mathrm{~mL}, \sim 0.07 \mathrm{M})$ in acetone at room temperature. The yellow color of dimethyldioxirane disappeared immediately. After evaporation of acetone, the residue was treated immediately with $10 \mathrm{~mL}$ of $2.0 \mathrm{M}$ allylmagnesium chloride as below.

\section{Typical procedure for the ring opening of epoxides with Grignard reagents-}

To a solution of epoxide $(2 \mathrm{mmol})$ in anhydrous THF $(15 \mathrm{~mL})$ was added dropwise a solution of Grignard reagent $\left(10 \mathrm{mmol}, 5\right.$ equiv) over two minutes at $0{ }^{\circ} \mathrm{C}$. The reaction mixture was warmed slowly to room temperature, then stirred overnight. The reaction mixture was partitioned between saturated aqueous $\mathrm{NH}_{4} \mathrm{Cl}$ and ether. The combined organic extract was dried $\left(\mathrm{Na}_{2} \mathrm{SO}_{4}\right)$ and concentrated, and the residue was chromatographed.

\section{Alcohol (6b)}

Clear oil (58\% yield), TLC $R_{f}=0.46$ (PE: acetone, $\left.3: 1\right), R_{f}=0.43$ for the minor isomer $\mathbf{5 f}$. IR (neat, $\mathrm{cm}^{-1}$ ): 3456 (s), 2935 (s), 2863 (s), 1638 (w), 1514 (m), $1454(\mathrm{~m}), 1239$ (m); ${ }^{1} \mathrm{H}$ NMR $\left(400 \mathrm{MHz}, \mathrm{CDCl}_{3}\right) \delta$ 7.15-7.30 (m, 4H), $5.23(\mathrm{~m}, 1 \mathrm{H}), 4.92(\mathrm{~d}, \mathrm{~J}=30.4 \mathrm{~Hz}, 1 \mathrm{H}), 4.89$ $(\mathrm{d}, \mathrm{J}=23.2 \mathrm{~Hz}, 1 \mathrm{H}), 4.02(\mathrm{t}, \mathrm{J}=3.2 \mathrm{~Hz}, 1 \mathrm{H}), 2.34-2.64(\mathrm{~m}, 2 \mathrm{H}), 2.34(\mathrm{~s}, 3 \mathrm{H}), 1.45-2.10(\mathrm{~m}$, $9 \mathrm{H}) ;{ }^{13} \mathrm{C}$ NMR $\left(\mathrm{CDCl}_{3}, 100 \mathrm{MHz}\right) \delta$ u: $141.9,135.9,117.1,45.2,41.3,28.3,27.0,21.2$, 20.1; d: 134.7, 129.5, 127.0, 74.1, 21.1; HRMS calcd for $\mathrm{C}_{16} \mathrm{H}_{21}\left(\mathrm{M}+\mathrm{H}-\mathrm{H}_{2} \mathrm{O}\right) 213.1643$, found 213.1644 .

\section{Alkene (10)}

To a Grignard reagent prepared from $\mathrm{Mg}(1.82 \mathrm{~g}, 75.0 \mathrm{mmol})$ and 4-bromoveratrole (13.0 g, $60.0 \mathrm{mmol}$ ) in $40 \mathrm{~mL}$ anhydrous THF at $0{ }^{\circ} \mathrm{C}$ was added 1,4-dioxaspiro[4,5]decan-8-one 8 $(7.80 \mathrm{~g}, 50.0 \mathrm{mmol})$ in $20 \mathrm{~mL}$ anhydrous THF over $5 \mathrm{~min}$ under nitrogen. After stirring at 0 ${ }^{\circ} \mathrm{C}$ for $30 \mathrm{~min}$, then ambient temperature for $2 \mathrm{~h}$, the reaction mixture was partitioned between ether and saturated aqueous $\mathrm{NH}_{4} \mathrm{Cl}$. The combined organic extract was dried $\left(\mathrm{Na}_{2} \mathrm{SO}_{4}\right)$ and concentrated. To the residue was added benzene $(200 \mathrm{~mL})$, ethylene glycol $(30 \mathrm{~mL})$ and $p$-toluenesulfonic acid $(10 \mathrm{mg})$. A Dean-Stark water separation apparatus was set up, and the solution was heated to reflux until about $0.9 \mathrm{~mL}$ of water had been collected. The reaction mixture was partitioned between ether and saturated aqueous $\mathrm{NaHCO}_{3}$. The organic extract was dried $\left(\mathrm{Na}_{2} \mathrm{SO}_{4}\right)$ and evaporated, and the residue was chromatographed to give $8.86 \mathrm{~g}$ of alkene $\mathbf{1 0}(64 \%$ yield from $\mathbf{8})$ as white solid: $\mathrm{mp}=76-78{ }^{\circ} \mathrm{C}\left(\right.$ lit. $^{10}=77.5-$ $\left.78{ }^{\circ} \mathrm{C}\right) .{ }^{1} \mathrm{H}$ NMR $\left(400 \mathrm{MHz}, \mathrm{CDCl}_{3}\right) \delta 6.80-6.95(\mathrm{~m}, 3 \mathrm{H}), 5.91(\mathrm{~m}, 1 \mathrm{H}), 4.01(\mathrm{~s}, 4 \mathrm{H}), 3.88$ (s, 3H), 3.87 (s, 3H), $2.64(\mathrm{~m}, 2 \mathrm{H}), 2.46(\mathrm{br}, 2 \mathrm{H}), 1.90(\mathrm{t}, \mathrm{J}=6.4 \mathrm{~Hz}, 2 \mathrm{H})$.

\section{Alcohol (6d)}

To a $500 \mathrm{~mL}$ three -necked flask was added alkene $\mathbf{1 0}(2.31 \mathrm{~g}, 8.37 \mathrm{mmol}), 85 \mathrm{~mL}$ of a $2: 1$ mixture of dimethoxymethane and acetonitrile, $50 \mathrm{~mL}$ of potassium carbonate-acetic acid buffer solution, tetrabutylammonium hydrogen sulfate $(63 \mathrm{mg})$ and Shi's chiral ketone $(0.756 \mathrm{~g}, 2.93 \mathrm{mmol})$. The flask was connected to two dropping funnels, one charged with a solution of oxone (7.72 g, $12.6 \mathrm{mmol})$ in $30 \mathrm{~mL}$ of aqueous $4 \times 10^{-4} \mathrm{Na}_{2}$ EDTA and the other charged with $30 \mathrm{~mL}$ of $1.47 \mathrm{M}$ aqueous $\mathrm{KOH}$. The two solutions were added dropwise at the same rate over $65 \mathrm{~min}$ to the cold reaction mixture which was stirred vigorously at $0{ }^{\circ} \mathrm{C}$. After $3 \mathrm{~h}$, ether $(100 \mathrm{~mL})$ was added. The biphasic mixture was partitioned between ether and, sequentially, water and brine. The organic extract was dried $\left(\mathrm{Na}_{2} \mathrm{SO}_{4}\right)$ and concentrated. The residue was dissolved in $50 \mathrm{~mL}$ of anhydrous THF. To this solution 
allylmagnesium chloride $(21 \mathrm{~mL}, 2.0 \mathrm{M})$ was added dropwise over 5 min at $0{ }^{\circ} \mathrm{C}$ under $\mathrm{N}_{2}$. The reaction solution was warmed slowly from $0{ }^{\circ} \mathrm{C}$ to room temperature, then maintained at ambient temperature overnight. The reaction mixture was partitioned between saturated aqueous $\mathrm{NH}_{4} \mathrm{Cl}$ and ether. The combined organic extract was dried over $\mathrm{Na}_{2} \mathrm{SO}_{4}$ and concentrated in vacuo. The residue was chromatographed to give $2.04 \mathrm{~g}$ of alcohol $\mathbf{6 d}$ (73\% yield from 10) as a colorless oil. TLC $R_{f}=0.56$ (ether), $[\alpha]^{22} \mathrm{D}=+37.3(\mathrm{c} 1.6, \mathrm{THF})$; IR (neat, $\mathrm{cm}^{-1}$ ): 3514 (s), 2954 (s), 1637 (w), 1587 (w), $1518(\mathrm{~s}), 1464(\mathrm{~m}), 1256(\mathrm{~s}), 1150(\mathrm{~m})$, $1028(\mathrm{~m}) ;{ }^{1} \mathrm{H}$ NMR $\left(400 \mathrm{MHz}, \mathrm{CDCl}_{3}\right) \delta$ 6.83-6.95 (m, 3H), $5.28(\mathrm{~m}, 1 \mathrm{H}), 4.96(\mathrm{~d}, \mathrm{~J}=23.6$ $\mathrm{Hz}, 1 \mathrm{H}), 4.93(\mathrm{~d}, \mathrm{~J}=17.2 \mathrm{~Hz}, 1 \mathrm{H}), 4.16(\mathrm{t}, \mathrm{J}=3.6 \mathrm{~Hz}, 1 \mathrm{H}), 3.97(\mathrm{~m}, 4 \mathrm{H}), 3.88(\mathrm{~s}, 3 \mathrm{H}), 3.86$ $(\mathrm{s}, 3 \mathrm{H}), 3.08(\mathrm{~d}, \mathrm{~J}=8.0 \mathrm{~Hz}, 1 \mathrm{H}), 1.65-2.50(\mathrm{~m}, 8 \mathrm{H}) ;{ }^{13} \mathrm{C} \mathrm{NMR}\left(\mathrm{CDCl}_{3}, 100 \mathrm{MHz}\right) \delta \mathrm{u}: 148.6$, 147.1, 137.2, 117.3, 108.7, 64.6, 64.1, 44.6, 41.6, 36.7, 30.5, 25.2; d: 134.3, 119.1, 110.8, 110.3, 74.3, 55.8, 55.7; HRMS calcd for $\mathrm{C}_{19} \mathrm{H}_{26} \mathrm{O}_{5}\left(\mathrm{M}^{+}\right) 334.1780$, found 334.1774.

\section{Enone (11)}

To a solution of alcohol $\mathbf{6 d}(0.90 \mathrm{~g}, 2.7 \mathrm{mmol})$ in $10 \mathrm{~mL}$ of THF was added $10 \%$ aqueous $\mathrm{HCl}(4 \mathrm{~mL})$. After heating to reflux for $1 \mathrm{~h}$, the reaction mixture was partitioned between saturated aqueous $\mathrm{Na}_{2} \mathrm{CO}_{3}$ and $\mathrm{CH}_{2} \mathrm{Cl}_{2}$. The combined $\mathrm{CH}_{2} \mathrm{Cl}_{2}$ extract was dried $\left(\mathrm{Na}_{2} \mathrm{SO}_{4}\right)$ and concentrated in vacuo. The residue was chromatographed to give $0.67 \mathrm{~g}$ of enone 11 (92\% yield) as a colorless oil, TLC $R_{f}=0.69$ (PE:EtOAc $\left.=1: 1\right),[\alpha]^{22} \mathrm{D}=-107.6(\mathrm{c} 0.8$, THF); IR (neat, $\mathrm{cm}^{-1}$ ): 2935 (m), 1682 (s), 1639 (w), 1603 (w), 1518 (s), 1464 (m), 1255 (s), $1148(\mathrm{~m}), 1027(\mathrm{~m}) ;{ }^{1} \mathrm{H}$ NMR $\left(400 \mathrm{MHz}, \mathrm{CDCl}_{3}\right) \delta 7.07(\mathrm{~d}, \mathrm{~J}=10.0 \mathrm{~Hz}, 1 \mathrm{H}), 6.86(\mathrm{~s}$, $3 \mathrm{H}), 6.17(\mathrm{~d}, \mathrm{~J}=10.0 \mathrm{~Hz}, 1 \mathrm{H}), 5.56(\mathrm{~m}, 1 \mathrm{H}), 5.11(\mathrm{~d}, \mathrm{~J}=15.2 \mathrm{~Hz}, 1 \mathrm{H}), 5.08(\mathrm{~d}, \mathrm{~J}=8.4 \mathrm{~Hz}$, $1 \mathrm{H}), 3.88(\mathrm{~s}, 3 \mathrm{H}), 3.87(\mathrm{~s}, 3 \mathrm{H}), 2.15-2.73(\mathrm{~m}, 6 \mathrm{H}) ;{ }^{13} \mathrm{C} \mathrm{NMR}\left(\mathrm{CDCl}_{3}, 90 \mathrm{MHz}\right) \delta$ u: 199.6, 149.1, 148.0, 135.4, 119.0, 46.3, 43.6, 36.1, 34.5; d: 155.3, 133.6, 129.5, 119.4, 111.1, 110.2, 56.1, 55.9; HRMS calcd for $\mathrm{C}_{17} \mathrm{H}_{21} \mathrm{O}_{3}(\mathrm{M}+\mathrm{H})$ 273.1491, found 273.1490.

\section{Diol (12)}

To a solution of enone $11(0.663 \mathrm{~g}, 2.43 \mathrm{mmol})$ in HPLC grade methanol $(20 \mathrm{~mL})$ was added an indicator amount of Sudan III. After purging with $\mathrm{O}_{2}$ at $-78{ }^{\circ} \mathrm{C}$ for $2 \mathrm{~min}, \mathrm{O}_{3}$ was bubbled through the solution until the red color of Sudan III disappeared. The reaction was purged with $\mathrm{N}_{2}$ for $5 \mathrm{~min}$, then $\mathrm{CeCl}_{3} \cdot 7 \mathrm{H}_{2} \mathrm{O}^{8}(1.00 \mathrm{~g}, 2.68 \mathrm{mmol})$ was added. The reaction mixture was cooled with an ice-water bath, then $\mathrm{NaBH}_{4}(554 \mathrm{mg}, 14.6 \mathrm{mmol})$ was added in three portions over $5 \mathrm{~min}$. After an additional $30 \mathrm{~min}, \mathrm{NaBH}_{4}(92 \mathrm{mg}, 2.43 \mathrm{mmol})$ was added followed by stirring for another $30 \mathrm{~min}$. The latter procedure was repeated once more. The reaction mixture was partitioned between water and $\mathrm{CH}_{2} \mathrm{Cl}_{2}$. The combined organic extract was dried $\left(\mathrm{Na}_{2} \mathrm{SO}_{4}\right)$ and concentrated, and the residue was chromatographed to give $0.474 \mathrm{~g}$ of diol 11 (4:1 mixture of two diasteromers, 73\% yield) as a colorless oil. TLC $R_{f}=$ 0.18 (wet ether), $[\alpha]^{22} \mathrm{D}=-23.5$ (c 0.55, THF); IR (neat, $\mathrm{cm}^{-1}$ ): 3390 (b), 2941 (s), 1715 (m), $1520(\mathrm{~s}), 1260(\mathrm{~s}), 1026$ (s); ${ }^{1} \mathrm{H}$ NMR $\left(400 \mathrm{MHz}\right.$, major isomer, $\left.\mathrm{CDCl}_{3}\right) \delta 6.78-6.82$ $(\mathrm{m}, 3 \mathrm{H}), 5.86-5.95(\mathrm{~m}, 2 \mathrm{H}), 4.21(\mathrm{~m}, 1 \mathrm{H}), 3.86(\mathrm{~s}, 3 \mathrm{H}), 3.85(\mathrm{~s}, 3 \mathrm{H}), 3.47-3.58(\mathrm{~m}, 2 \mathrm{H})$, 1.62-2.07 (m, 7H); ${ }^{13} \mathrm{C} \mathrm{NMR}\left(\mathrm{CDCl}_{3}\right.$, major isomer, $\left.90 \mathrm{MHz}\right) \delta$ u: 148.8, 147.4, 139.1, 59.7, 45.0, 41.8, 35.0, 28.9; d: 134.9, 131.7, 119.4, 110.9, 110.5, 67.2, 56.1, 56.0; ${ }^{1} \mathrm{H}$ NMR (400 MHz, minor isomer, $\left.\mathrm{CDCl}_{3}\right) \delta 6.80-6.82(\mathrm{~m}, 3 \mathrm{H}), 5.60-6.06(\mathrm{~m}, 2 \mathrm{H}), 4.13(\mathrm{~m}, 1 \mathrm{H})$, $3.87(\mathrm{~s}, 3 \mathrm{H}), 3.85(\mathrm{~s}, 3 \mathrm{H}), 3.55-3.65(\mathrm{~m}, 2 \mathrm{H}), 1.62-2.10(\mathrm{~m}, 7 \mathrm{H}) ;{ }^{13} \mathrm{C} \mathrm{NMR}\left(\mathrm{CDCl}_{3}\right.$, minor isomer, $90 \mathrm{MHz}) \delta \mathrm{u}: 148.9,147.5,139.0,59.7,44.7,41.9,32.6,28.5$; d: 136.5, 130.0, 119.0, 111.1, 110.3, 64.6, 56.1, 56.0; HRMS calcd for $\mathrm{C}_{16} \mathrm{H}_{22} \mathrm{O}_{4} \mathrm{Na}(\mathrm{M}+\mathrm{Na}) 301.1416$, found 301.1412 .

\section{Tosylate (13)}

To a solution of diol 12 (major isomer, $133 \mathrm{mg}, 0.478 \mathrm{mmol}$ ) in $5 \mathrm{~mL}$ of anhydrous $\mathrm{CH}_{2} \mathrm{Cl}_{2}$ was added triethylamine $(145 \mathrm{mg}, 1.44 \mathrm{mmol})$ and tosyl chloride $(91.2 \mathrm{mg}, 0.478 \mathrm{mmol})$. After stirring under $\mathrm{N}_{2}$ at ambient temperature for $16 \mathrm{~h}$, the reaction mixture was partitioned 
between saturated aqueous $\mathrm{Na}_{2} \mathrm{CO}_{3}$ and $\mathrm{CH}_{2} \mathrm{Cl}_{2}$. The combined organic extract was dried $\left(\mathrm{Na}_{2} \mathrm{SO}_{4}\right)$ and concentrated. The crude product was chromatographed to give $0.186 \mathrm{~g}$ of tosylate 13 (90\% yield) as a clear oil. TLC $R_{f}=0.34$ (ether), $[\alpha]^{22} \mathrm{D}=-11.4(\mathrm{c}=0.35$, THF); IR (neat, $\mathrm{cm}^{-1}$ ): 3390 (b), 2938 (s), 1598 (s), 1464 (m), 1359 (s), 1252 (s), 1176 (s); ${ }^{1} \mathrm{H}$ NMR $\left(400 \mathrm{MHz}, \mathrm{CDCl}_{3}\right) \delta 7.69(\mathrm{~d}, \mathrm{~J}=8.0 \mathrm{~Hz}, 2 \mathrm{H}), 7.30(\mathrm{~d}, \mathrm{~J}=8.0 \mathrm{~Hz}, 2 \mathrm{H}), 6.75(\mathrm{br}, 3 \mathrm{H})$, 5.90 (dd, J=10.0 Hz, 1.2 Hz, 1H), $5.78(\mathrm{~d}, \mathrm{~J}=10.0 \mathrm{~Hz}, 1 \mathrm{H}), 4.19$ (br, 1H), 3.81-3.98 (m, 2H), $3.85(\mathrm{~s}, 3 \mathrm{H}), 3.84(\mathrm{~s}, 3 \mathrm{H}), 2.44(\mathrm{~s}, 3 \mathrm{H}), 1.67-2.17(\mathrm{~m}, 7 \mathrm{H}) ;{ }^{13} \mathrm{C} \mathrm{NMR}\left(\mathrm{CDCl}_{3}, 100 \mathrm{MHz}\right) \delta$ u: 149.0, 147.7, 144.9, 137.8, 133.2, 67.8, 41.8, 40.9, 35.0, 28.8; d: 133.7, 132.5, 129.9, 127.9, 119.4, 111.1, 110.3, 67.0, 56.1, 56.0, 21.8; HRMS calcd for $\mathrm{C}_{23} \mathrm{H}_{28} \mathrm{O}_{6} \mathrm{~S}\left(\mathrm{M}^{+}\right)$ 432.1607, found 432.1605 .

\section{(-)-Mesembrine (1)}

To a sealed thick-walled reaction flask was added tosylate $\mathbf{1 3}(144 \mathrm{mg}, 0.33 \mathrm{mmol})$, methylamine $(2.0 \mathrm{~mL}, 40 \%$ in water), and THF $(4.0 \mathrm{~mL})$. The reaction mixture was heated at $65^{\circ} \mathrm{C}$ for $1 \mathrm{~h}$, then concentrated in vacuo, and the residual oil was dissolved in $\mathrm{CH}_{2} \mathrm{Cl}_{2}$ $(15 \mathrm{~mL})$. To this solution activated $\mathrm{MnO}_{2}(220 \mathrm{mg}, 2.53 \mathrm{mmol})$ was added, and the mixture was stirred at $\mathrm{rt}$ for $3 \mathrm{~h}$. After filtration through a thin layer of Celite, the filtrate was concentrated and the residue was chromatographed to give $58.8 \mathrm{mg}$ of (-)-mesembrine (61\% yield) as a colorless oil. TLC $R_{f}=0.21$ (wet ether), $[\alpha]^{22} \mathrm{D}=-55.4(\mathrm{c}=0.54, \mathrm{MeOH})$; IR (neat, $\mathrm{cm}^{-1}$ ): $2944(\mathrm{~m}), 1716(\mathrm{~s}), 1520(\mathrm{~s}), 1454(\mathrm{~m}), 1253(\mathrm{~s}), 1148(\mathrm{~m}), 1027(\mathrm{~m}) ;{ }^{1} \mathrm{H}$ $\operatorname{NMR}\left(400 \mathrm{MHz}, \mathrm{CDCl}_{3}\right) \delta 6.93(\mathrm{dd}, \mathrm{J}=8.4,2.4 \mathrm{~Hz}, 1 \mathrm{H}), 6.90(\mathrm{~d}, \mathrm{~J}=2.0 \mathrm{~Hz}, 1 \mathrm{H}), 6.84(\mathrm{~d}$, $\mathrm{J}=8.4 \mathrm{~Hz}, 1 \mathrm{H}), 3.90(\mathrm{~s}, 3 \mathrm{H}), 3.88(\mathrm{~s}, 3 \mathrm{H}), 3.13-3.17(\mathrm{~m}, 1 \mathrm{H}), 2.96(\mathrm{t}, \mathrm{J}=3.6 \mathrm{~Hz}, 1 \mathrm{H}), 2.61$ $(\mathrm{m}, 2 \mathrm{H}), 2.35-2.46(\mathrm{~m}, 2 \mathrm{H}), 2.33(\mathrm{~s}, 3 \mathrm{H}), 2.04-2.26(\mathrm{~m}, 5 \mathrm{H}) ;{ }^{13} \mathrm{C} \mathrm{NMR}\left(\mathrm{CDCl}_{3}, 90 \mathrm{MHz}\right) \delta$ u: $211.5,149.3,147.8,140.4,55.1,47.8,40.8,39.1,36.4,35.5$; d: 118.2, 111.3, 110.3, 70.6, 56.2, 56.1, 40.3; HRMS calcd for $\mathrm{C}_{17} \mathrm{H}_{23} \mathrm{NO}_{3}\left(\mathrm{M}^{+}\right)$289.1678, found 289.1671 .

\section{Supplementary Material}

Refer to Web version on PubMed Central for supplementary material.

\section{Acknowledgments}

We thank the NIH (GM60287) for financial support of this study, and DSM for a gift of Shi ketone.

\section{REFERENCES}

1. For reviews on the enantioselective construction of quaternary stereogenic centers, see: (a) Martin SF. Tetrahedron. 1980; 36:419. (b) Fuji K. Chem. Rev. 1993; 93:2037. (c) Corey EJ, Guzman-Perez A. Angew. Chem. Int. Ed. 1998; 37:388. (d) Christoffers J, Mann A. Angew. Chem. Int. Ed. 2001; 40:4591. (e) Dennisova I, Barriault L. Tetrahedron. 2003; 59:10105. (f) Douglas CJ, Overman LE. Proc. Nat. Acad. Sci. 2004; 101:5363. [PubMed: 14724294]

2. For the isolation and structural determination of (-)-mesembrine, see (a) Popelak A, Haack E, Lettenbauer G, Spingler H. Naturwissenschaften. 1960; 47:156. (b) Smith E, Hosansky N, Shamma M, Moss JB. Chem. \& Ind. 1961:402.

3. For leading references to previous enantioselective syntheses of (-)-mesembrine, see (a) Takano S, Imamura Y, Ogasawara K. Tetrahedron Lett. 1981; 22:4479. (b) Takano S, Samizu K, Ogasawara K. Chem. Lett. 1990:1239. (c) Fukumoto K, Tanabe T, Nemoto H. J. Org. Chem. 1995; 60:6785. (d) Mori M, Kuroda S, Zhang C, Sato Y. J. Org. Chem. 1997; 62:3263. [PubMed: 11671713] (e) Denmark SE, Marcin LR. J. Org. Chem. 1997; 62:1675. (f) Langlois Y, Dalko PI, Brun V. Tetrahedron Lett. 1998; 39:8979. (g) Ogasawara K, Yamada O. Tetrahedron Lett. 1998; 39:7747. (h) Taber DF, Neubert TD. J. Org. Chem. 2001; 66:143. [PubMed: 11429891] For leading references to previous enantioselective syntheses of (+)-mesembrine see (i) Meyers AI, Hanreich R, Wanner KT. J. Am. Chem. Soc. 1985; 107:7776. (j) Kosugi H, Miura Y, Kanna H, Uda H. Tetrahedron Asymmetry. 1993; 4:1409. 
4. For leading references to the synthesis of racemic mesembrine, see (a) Chavan SP, Khobragade DA, Pathak AB, Kalkote UR. Tetrahedron Lett. 2004; 45:5263. (b) Kulkarni MG, Rasne RM, Davawala SI, Doke AK. Tetrahedron Lett. 2002; 43:2297. (c) Rigby JH, Dong W. Organic Lett. 2000; 2:1673.

5. For the cultural background and some limited activity trials of Sceletium tortuosum, see Smith MT, Crouch N, Gericke N, Hirst M. J. Ethnopharm. 1996; 50:119.

6. For the in vitro and in vivo activity of (-)-mesembrine, see Gericke NP, VanWyk B-E. PCT Int. Appl. 1997 WO 9746234 CAN 128:80030.

7. Taber DF, He Y, Xu M. J. Am. Chem. Soc. 2004; 126:13900. [PubMed: 15506732]

8. For reports of the opening of arene-activated trisubstituted epoxides at the more substituted carbon to establish a quaternary center, see: (a) Gerteisen TJ, Kleinfelter DC. J. Org. Chem. 1971; 36:3255. (b) Krause N, Seebach D. Chem. Ber. 1988; 121:1315. (c) Kauffmann T, Neiteler C, Robbe S. Chem. Ber. 1992; 125:2409. (d) Jansen J, Knopp M, Amberg W, Bernard H, Koser S, Müller S, Münster I, Pfeiffer T, Riechers H. Org. Process Research \& Development. 2001; 5:16.

9. For the preparation of the alkenes corresponding to $\mathbf{5 a}$ and $\mathbf{5 c}$, see (a) Balsamo A, Crotti P, Macchia B, Macchia F. Tetrahedron. 1973; 29:2183. (b) For the preparation of the alkenes corresponding to 5b and 5c, see Doan L, Bradley K, Gerdes S, Whalen DL. J. Org. Chem. 1999; 64:6227.

10. For the preparation of 5d, see Hoshino O, Sawaki S, Shimamura N, Onodera A, Umezawa B. Chem. Pharm. Bull. 1987; 35:2734.

11. We found that the sensitive epoxides could be prepared efficiently from the alkenes with dimethyl dioxirane: Murray RW, Singh M. Org. Syn. 1997; 74:91.

12. Newbold RC, Shih TL, Mrozik H, Fisher MH. Tetrahedron Lett. 1993; 34:3825.

13. Tu Y, Wang Z-X, Shi Y. J. Am. Chem. Soc. 1996; 118:9806.

14. Sousa JA, Bluhm AC. J. Org. Chem. 1960; 25:108.

15. Gemal AL, Luche J-L. J. Am. Chem. Soc. 1981; 103:5454.

16. (a) Trost BM, Pissot-Soldermann C, Chen I, Schroeder GM. J. Am. Chem. Soc. 2004; 126:4480. [PubMed: 15070341] (b) Trost BM, Xu J. J. Am. Chem. Soc. 2005; 127:2846. [PubMed: 15740108]

17. (a) Aahman J, Wolfe JP, Troutman MV, Palucki M, Buchwald SL. J. Am. Chem. Soc. 1998; 120:1918.(b) Hamada T, Chieffi A, Ahman J, Buchwald SL. J. Am. Chem. Soc. 2002; 124:1261. [PubMed: 11841295] 


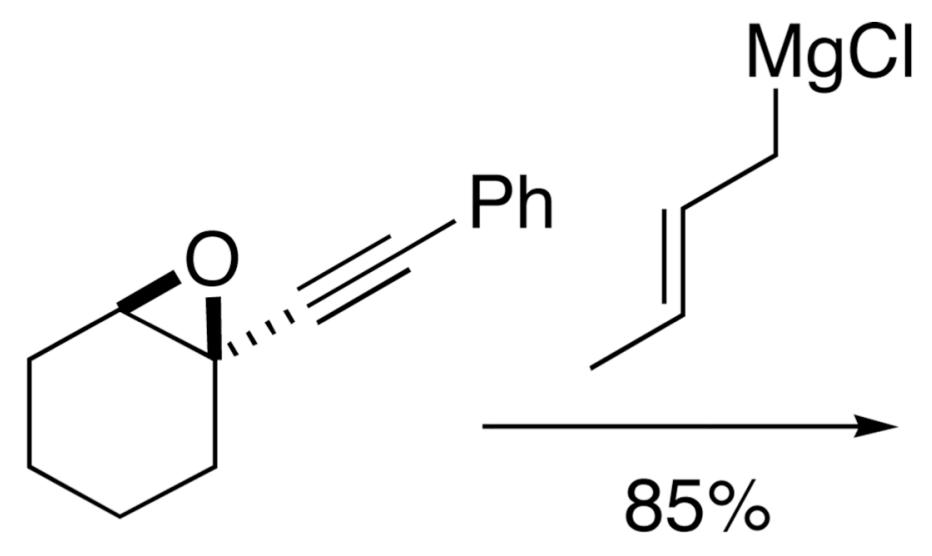

3

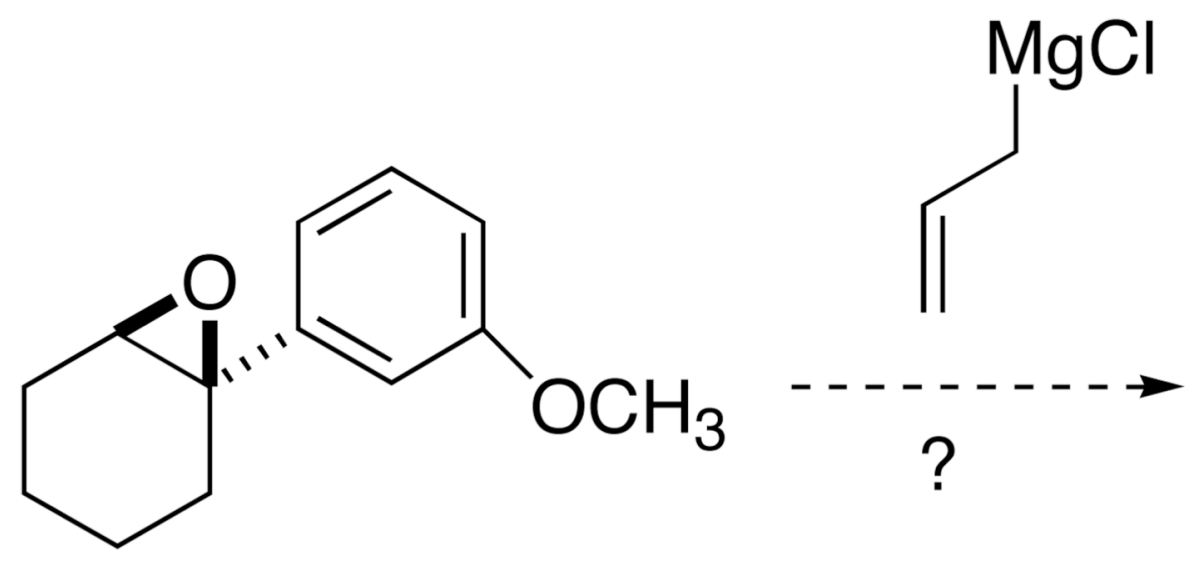

$5 a$

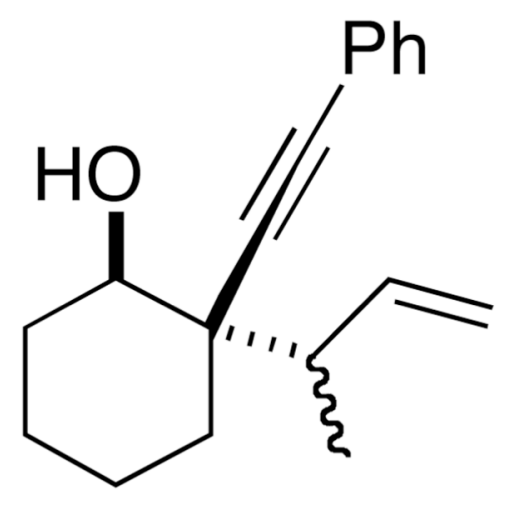

4

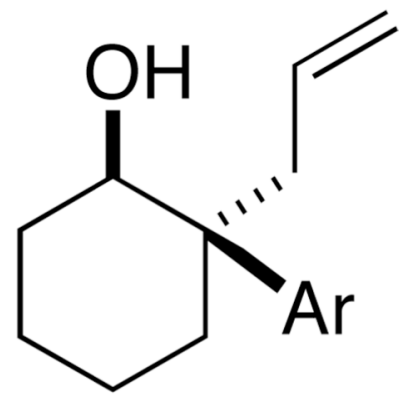

$6 a$

Scheme 1. 
<smiles>COc1ccc(C2=CCC3(CC2)OCCO3)cc1OC(=O)[O-]</smiles><smiles>C=CC[C@]1(c2ccc(OC)c(OC)c2)C=CC(=O)CC1</smiles><smiles>COc1ccc([C@]2(CCOC(F)(F)F)C=C[C@@H](O)CC2)cc1OC</smiles><smiles>COc1ccc(C23CCC(=O)CC2N(C)CC3)cc1OC</smiles>

(-)-Mesembrine 1

Scheme $2^{a}$

a (a) THF, $0{ }^{\circ} \mathrm{C}$ to $20^{\circ} \mathrm{C}$, overnight; (b) benzene, PTSA (cat.), ethylene glycol (excess), reflux; (c) Shi's catalyst, DME-acetonitrile- $\mathrm{H}_{2} \mathrm{O}, 0{ }^{\circ} \mathrm{C}, 4 \mathrm{~h}$; (d) allylmagnesium chloride, THF, $0{ }^{\circ} \mathrm{C}$ to $20{ }^{\circ} \mathrm{C}$, overnight; (e) $10 \%$ aqueous $\mathrm{HCl}$, THF, reflux, $1 \mathrm{~h}$; (f) $\mathrm{O}_{3}, \mathrm{MeOH},-78$ ${ }^{\circ} \mathrm{C} ; \mathrm{CeCl}_{3} \cdot 7 \mathrm{H}_{2} \mathrm{O}$ (1.0 equiv), $\mathrm{NaBH}_{4}$ (8.0 equiv), $0^{\circ} \mathrm{C}$; (g) $\mathrm{TsCl}$ (1 equiv), $\mathrm{Et}_{3} \mathrm{~N}, \mathrm{CH}_{2} \mathrm{Cl}_{2}, 20$ ${ }^{\circ} \mathrm{C}$, overnight; (h) $40 \%$ aqueous $\mathrm{CH}_{3} \mathrm{NH}_{2}$, THF, $65^{\circ} \mathrm{C}, 1 \mathrm{~h}$; (i) $\mathrm{MnO}_{2}, \mathrm{CH}_{2} \mathrm{Cl}_{2}, 20{ }^{\circ} \mathrm{C}, 3 \mathrm{~h}$. 
Table 1

Opening of Arene-Substituted Cyclic Epoxides

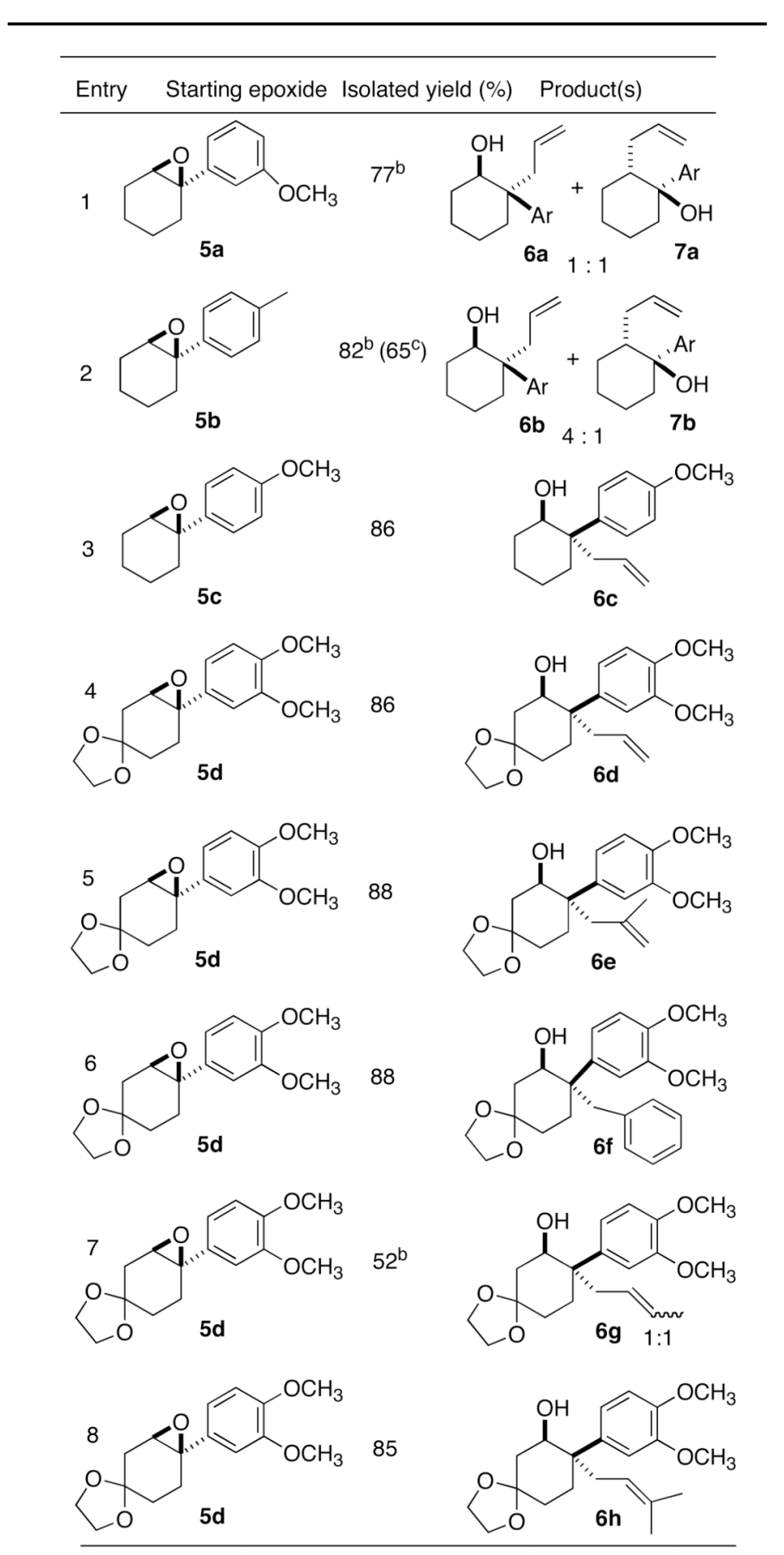

${ }^{a}$ Ratio of two isomers was determined by ${ }^{1} \mathrm{H}$ NMR.

${ }^{b}$ Combined yields of two isomers.

$c_{\text {Isolated yield of } \mathbf{6 b}}$ 